\title{
EXPLICIT SOLUTIONS OF FISHER'S EQUATION WITH THREE ZEROS
}

\author{
M.F.K. ABUR-ROBB \\ Department of Mathematics \\ The University \\ Al-Ain \\ United Arab Emirates \\ (Received January 24, 1989)
}

ABSTRACT. Explicit traveling wave solutions of Fisher's equation with three simple zeros $u_{t}=u_{x x}+u(1-u)(u-a)$, a $\varepsilon(0,1)$, are obtained for the wave speeds

$C= \pm \sqrt{2}(1 / 2-a)$ suggested by pure analytic considerations. Two types of solutions are obtained: one type is of a permanent wave form whereas the other is not.

KEY WORDS AND PHRASES. Nonlinear diffusion equation, Fisher's equation, and wave solutions 1980 AMS SUBJECT CLASSIFICATION CODES. 35K57

1. INTRODUCTION.

The scalar non-linear differential equation of reaction and diffusion

$$
u_{t}=u_{x x}+f(u)
$$

arises in many applications. The particular case $f(u)=u(1-u)$ was introduced by Fisher [1] in connection with "genetic waves" which are simply wave front solutions. Since fronts generally exist when $f$ has many zeros, equation (1.1) with this property is sometimes referred to as "Fisher's non-linear diffusion equation" (Fife [2]).

The case when $f$ has exactly one zero is trivial whereas the case of two simple zeros was originally proposed by Fisher [1] as a model for the propagation of a mutant gene with an advantageous selection intensity. Fisher's equation with $f(u)=u(1-u)$ occurs also in flame propagation, in the branching Brownian motion, and in nuclear reactor theory (Canosa [3]). The first known explicit traveling wave solution of the Fisher equation with two simple zeros was obtained by Ablowitz and Zeppetella [4] for the special wave speeds $C= \pm 5 / \sqrt{6}$.

In this paper we consider the interesting case when $f$ has exactly three zeros, the two outer ones stable, the inner one unstable, and all three simple. This occurs as a population genetic model, as well as in the study of transmission lines, combustion theory, and some degenerate cases of nerve signal propagation. Assume the 
two stable zeros are at $u=0$ and $u=1$, and the unstable one lies in between. A function $f(u)$ that fits this model would be

$$
f(u)=u(1-u)(u-a)=-a u+(1+a) u^{2}-u^{3}
$$

where a $\varepsilon(0,1)$. On substituting $u(x, t)=U(z)=U(x-C t)$ in $(1.1)$ and $(1.2)$, we obtain the traveling wave equation

$$
U^{\prime \prime}+C U^{\prime}-a U+(1+a) U^{2}-U^{3}=0
$$

where primes denote differentiation with respect to $z$.

\section{MAIN RESULTS.}

Our objective is to explore the possibility of explicit solutions for equation (1.3). In fact we discover by pure analytic considerations that equation (1.3) can have explicit solutions when the functional dependence of $C$ on $a$ is of the farm $C= \pm \sqrt{2}(1 / 2-a)$.

By assumption $u=0$ and $u=1$ are both stable rest points for the kinetic equation $u_{t}=f(u)$, so we are in the bistable case. Hence there is a unique solution $U((z), C)$ depending on $a$, of course. Let $g$ denote the functional dependence of $C$ on $a$, $C=g(a)$. To find this functional relationship we begin by determining what kind of pole a complex solution of $(1.3)$ can have. If $U(z) \sim K\left(z-z_{0}\right)^{-r}$, then the $U^{\prime \prime}$ and $U^{3}$ terms must balance. Hence

$$
U^{\prime \prime} \sim K(-r)(-r-1)\left(z-z_{0}\right)^{-r-2}=k^{3}\left(z-z_{0}\right)^{-3 r} \sim U^{3}
$$

This gives $r=1$ and $k^{2}=2$ for a non-trivial solution. If the solutions are of the Painlevé type, then it is necessary that any Laurent series representation have coefficlents well-defined by the differential equation. (A review of Painleve's work appears in Ince, [5]). Thus we look for a solution of the form

$$
U(z)=K / z+a_{0}+a_{1} z+a_{2} z+\ldots
$$

where $K^{2}=2$ and use of the translational invariance of (1.3) is made to drop the parameter $z_{0^{\circ}}$ When $(2.1)$ is substituted in (1.3) and coefficients of $z^{-2}, z^{-1}, z^{0}$, ...are successively equated to zero to get expressions for $a_{0}, a_{1}, a_{2}$ as functions of. $C$ and a. A problem arises when attempting to find $a_{3}$. Upon equating the coefficient of $z^{1}$ to zero we get, after somewhat tedious algebra, the equation

$$
(0) \cdot a_{3}+c^{2}\left[(1-2 a)^{2}-2 c^{2}\right]=0
$$


For the Laurent series representation to be valid, $C$ must satisfy equation (2.2) which gives $c=0, g_{1}(a), g_{2}(a)$ where $g_{1}=-g_{2}=\sqrt{2}(1 / 2-a)$. For $C=0$, explicit solutions are not feasible unless $a=1 / 2$. Solutions with $C=g_{2}(a)$ are treated by changing $x$ into $-x$. Thus, we consider only the case

$$
c=g_{1}(a)=\overline{\sqrt{2}}(1 / 2-a)
$$

On substituting (2.3) in (1.3), the latter equation becomes

$$
U^{\prime \prime}+\sqrt{2}(1 / 2-a) U^{\prime}-a U+(1+a) U^{2}-U^{3}=0
$$

or

$$
U^{\prime \prime}+\frac{1}{\sqrt{2}} U^{\prime}+U^{2}-U^{3}=a\left(\sqrt{2} U^{\prime}+U-U^{2}\right) \text {. }
$$

To solve equation (2.4) we assert that every solution $U(z)$ of (2.4), if it exists, must be valid for all a. Our assertion is true only if $U(z)$ satisfies the following equations:

$$
\begin{aligned}
& \overline{\sqrt{2}} U^{\prime}+U-U^{2}=0, \\
& U^{\prime \prime}+\frac{1}{\sqrt{2}} U^{\prime}+U^{2}-U^{3}=0 .
\end{aligned}
$$

clearly (2.5) can be solved directly leading to that solution of (2.6) satisfying the conditions of biological interest

$$
\mathrm{U}(-\infty)=1, \quad \mathrm{U}(\infty)=0
$$

In fact, equation (2.5) is a Bernoulli equation whose solution satisfying (2.7) is

$$
u_{1}(x, t)=U(x-C t)=U(z)=1 /\left(1+b e^{z / \sqrt{2}}\right) \text {, }
$$

where $b$ is an arbitrary constant that ought to be positive; otherwise, solutions with negative $b$ blow up for finite real $z$. That the solution (2.8) of (2.5) also satifies (2.6) can be demonstrated by a direct, straigtforward calculation.

Our solution (2.8) represents a traveling wave which can be thought of as connecting the two trivial solutions $u=0$ and $u=1$. It has a unique velocity $C$ and, except for translation, a permanent wave form as well.

Reflection in. $x$ yields a wave traveling in the opposite direction

$$
u_{1}(-x, t)=U(-x-C t), U(-\infty)=0, U(\infty)=1
$$


If $C \neq 0, a$ combination of $u_{1}$ and its reflection, yield a second solution in the form of a diverging structure. This solution, which is not of permanent form, may be represented by the function

$$
u_{2}(x, t)=\left\{\begin{array}{l}
u_{1}(x, t), C x>0, \\
u_{1}(-x, t), C x<0,
\end{array}\right.
$$

REFERENCES

1. FISHER, R.A., The Wave of Advance of an Advantageous Gene, Ann. Eugen., $\underline{7}$, (1936), 355-369.

2. FIFE, P.C., Asymptotic States for Equations of Reaction and Diffusion, Bull. Am. Math. Soc., 84 (1978), 693-726.

3. CANOSA, J., Diffusion in Nonlinear Multiplicative Media, J. Math. Phys., 10, (1969), 1862 .

4, ABLOWITZ, M. and ZEPPETELLA, A., Explicit Solutions of Fisher's Equation for a Special Wave Speed". Bull. Math. Biology, 41 (1979), 835-840.

5. INCE, E.I., Ordinary Differential Equations, New York: Dover, 1956). 


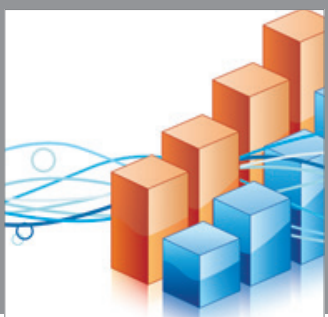

Advances in

Operations Research

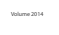

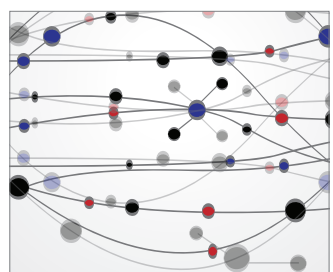

\section{The Scientific} World Journal
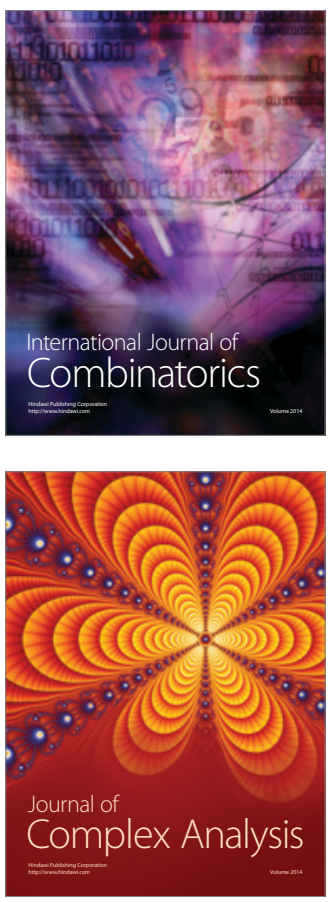

International Journal of

Mathematics and

Mathematical

Sciences
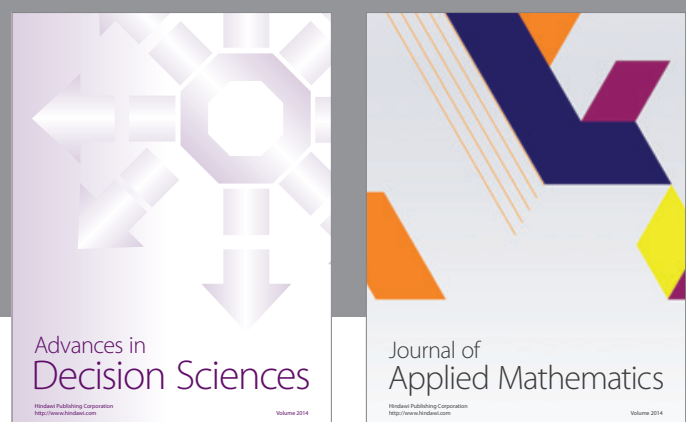

Journal of

Applied Mathematics
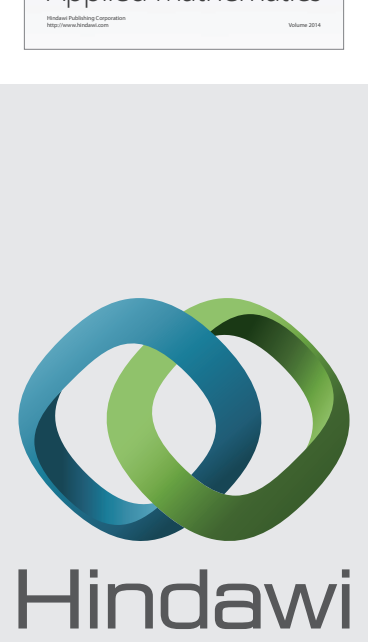

Submit your manuscripts at http://www.hindawi.com
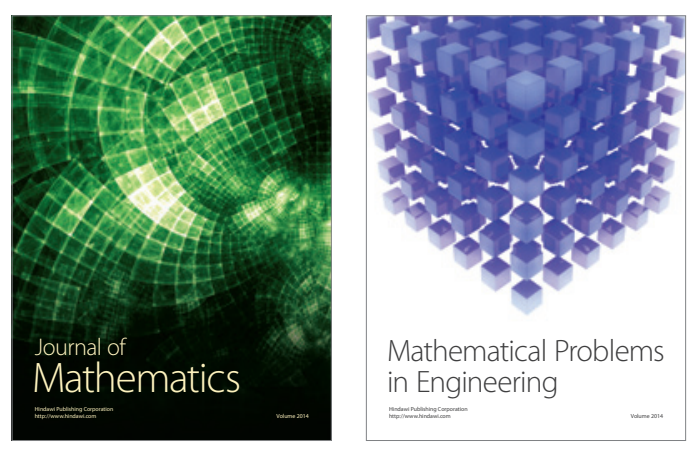

Mathematical Problems in Engineering
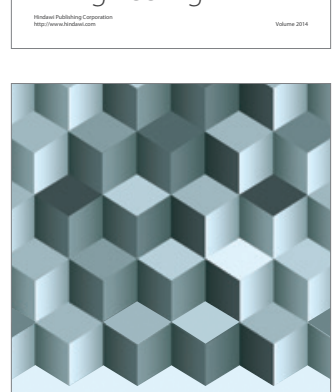

Journal of

Function Spaces
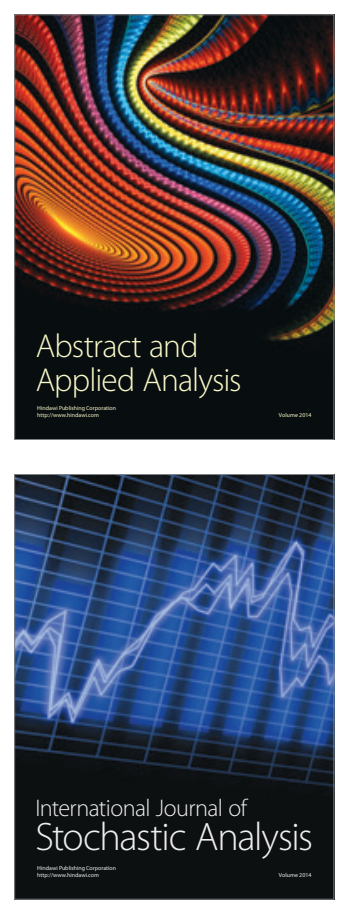

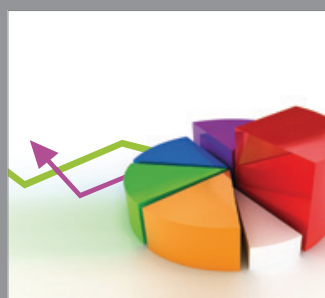

ournal of

Probability and Statistics

Promensencen
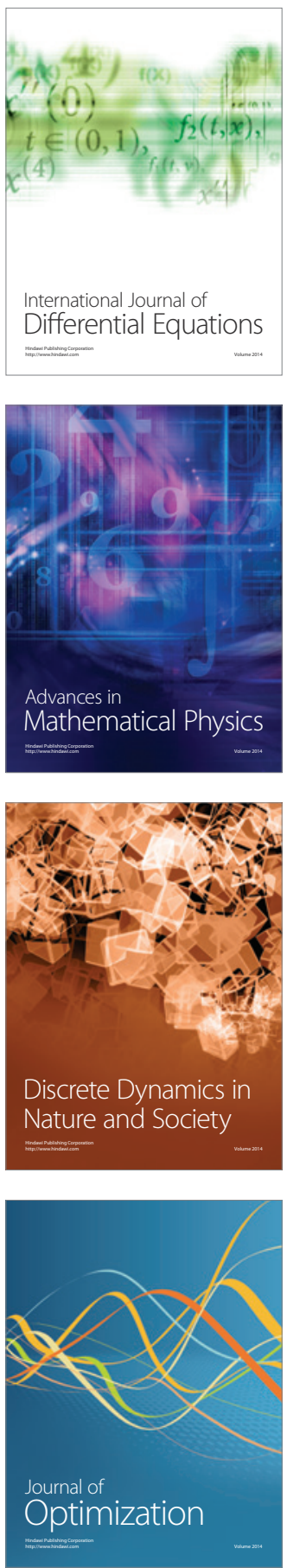